\title{
UK Renal Registry 11th Annual Report (December 2008): Appendix H Acronyms and abbreviations used in the Report
}

\author{
ACE (inhibitor) \\ APD \\ ARF \\ ASSIST \\ AVF \\ BAPN \\ BCG \\ $\mathrm{BCP}$ \\ BMI \\ $\mathrm{BOO}$ \\ BP \\ BTS \\ $\mathrm{CAB}$ \\ CABG \\ CAPD \\ CCL \\ CCPD \\ CI \\ CIC \\ CKD \\ CMMS (CMS) \\ COPD \\ CRF \\ CRP \\ CXR \\ DBP \\ DCCT \\ DFS \\ DM \\ $\mathrm{DoH}$ \\ DOPPS \\ DOQI \\ E\&W \\ EBPG \\ eGFR \\ ER \\ ERA \\ ERA-EDTA \\ EPO \\ EPR \\ ERF

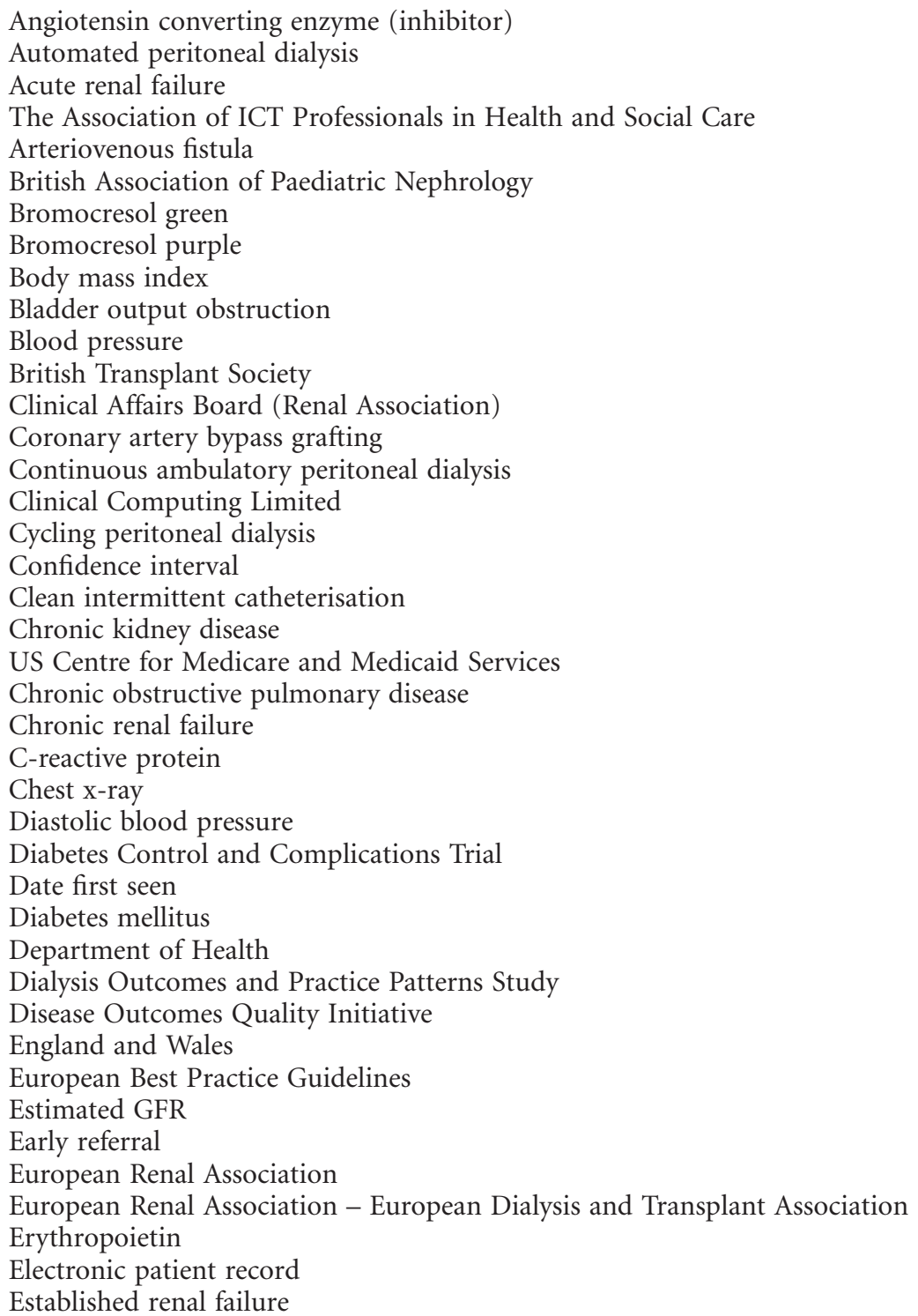

\section{KARGER}

Fax +4161306 1234 E-Mail karger@karger.ch www.karger.com
C 2009 S. Karger AG, Basel

$1160-2110 / 09 / 1115-0332 \$ 26.00 / 0$

Accessible online at: www.karger.com/nec
UK Renal Registry, Southmead Hospital, Southmead Road, Bristol BS10 5NB, UK

Email: renalreg@renalreg.com 


\begin{tabular}{|c|c|}
\hline ESA & Erythropoietin stimulating agent \\
\hline ESRD & End stage renal disease \\
\hline ESRF & End stage renal failure \\
\hline EWNI & England, Wales and Northern Ireland \\
\hline FSGS & Focal segmental glomerulosclerosis \\
\hline GFR & Glomerular filtration rate \\
\hline GN & Glomerulonephritis \\
\hline HA & Health Authority \\
\hline HbAlc & Glycated Haemoglobin \\
\hline HCAI-DCS & Healthcare Associated Infection Data Capture System \\
\hline HCFA & USA Health Care Finance Administration - now replaced by CMMS \\
\hline HD & Haemodialysis \\
\hline HDL & High-density lipoprotein \\
\hline $\mathrm{Hb}$ & Haemoglobin \\
\hline HLA & Human leucocyte antigen \\
\hline HQIP & Healthcare Quality Improvement Partnership \\
\hline HR & Hazard ratio \\
\hline ICNARC & National intensive care audit \\
\hline ICRS & Integrated care records system \\
\hline IHD & Ischaemic heart disease \\
\hline IDOPPS & International Dialysis Outcomes and Practice Patterns Study \\
\hline IFCC & International Federation of Clinical Chemistry \& Laboratory Medicine \\
\hline IM\&T & Information Management \& Technology \\
\hline IPD & Intermittent peritoneal dialysis \\
\hline iPTH & Intact parathyroid hormone \\
\hline ITU & Intensive therapy unit \\
\hline ISB & Information Standards Board \\
\hline KDOQI & Kidney Disease Outcomes Quality Initiative \\
\hline KM & Kaplan Meier \\
\hline LA & Local Authority \\
\hline LDL & Low-density lipoprotein \\
\hline LR & Late referral \\
\hline LSPs & Local service providers \\
\hline LV & Left ventricular \\
\hline LVH & Left ventricular hypertrophy \\
\hline MAP & Mean arterial blood pressure \\
\hline MDRD study & Modified Diet in Renal Disease study \\
\hline MDT & Multi-disciplinary team \\
\hline MESS & Mandatory Enhanced Surveillance System \\
\hline MI & Myocardial infarction \\
\hline MINAP & Myocardial infarction audit \\
\hline MRSA & Methicillin resistant Staphylococcal aureus \\
\hline NASP & National Application Service Providers \\
\hline NCRS & National Care Records Service \\
\hline $\mathrm{NeLH}$ & National electronic library for health \\
\hline NEQAS & UK National External Quality Assessment Scheme \\
\hline NFKPA & National Federation of Kidney Patients' Associations \\
\hline NHS & National Health Service \\
\hline NHID & National Health Informatics Development \\
\hline NHS BT & National Health Service Blood and Transplant \\
\hline NHSIA & NHS Information Agency \\
\hline NICE & National Institute of Clinical Excellence \\
\hline NPfIT & National Programme for Information Technology \\
\hline NSF & National service framework \\
\hline $\mathrm{OA}$ & Output area (census) \\
\hline OBSC & Output based specification contract \\
\hline ONS & Office of National Statistics \\
\hline PCT & Primary Care Trust \\
\hline $\mathrm{PD}$ & Peritoneal dialysis \\
\hline PIAG & Patient Information Advisory Group \\
\hline
\end{tabular}




\begin{tabular}{|c|c|}
\hline PKD & Polycystic kidney disease \\
\hline PMCP & Per million child population \\
\hline PMP & Per million population \\
\hline PP & Pulse pressure \\
\hline PTH & Parathyroid hormone \\
\hline PUV & Posterior urethral valves \\
\hline PVD & Peripheral vascular disease \\
\hline RA & Renal Association \\
\hline RNSF & Renal National Service Framework (or NSF) \\
\hline ROCR & Review of central information requirements \\
\hline RR & Relative risk \\
\hline RRDSS & Renal Registry data set specification \\
\hline RRT & Renal replacement therapy \\
\hline SARR & Standardised acceptance rate ratio \\
\hline SAS & Statistical Analysis System (statistical software used by the Registry \\
\hline SBP & Systolic blood pressure \\
\hline SD & Standard deviation \\
\hline SDS & Standard deviation score \\
\hline SDII & Renal Standards document - second edition \\
\hline SDIII & Renal Standards document - third edition \\
\hline SES & Socio-economic status \\
\hline SHARP & Study of Heart and Renal Protection \\
\hline SI & System International (units) \\
\hline SIRS & Study of Implementation of Renal Standards \\
\hline SMR & Standardised mortality ratios \\
\hline StHAs & Strategic health authorities \\
\hline SUS & Secondary uses service \\
\hline TOR & Take-on rate \\
\hline TSAT & Transferrin saturation \\
\hline UA & Unitary authorities \\
\hline UKCHIP & UK Council for Health Informatics Professions \\
\hline UKRR & UK Renal Registry \\
\hline UKT & UK Transplant \\
\hline USRDS & United States Renal Data System \\
\hline URR & Urea reduction ratio \\
\hline
\end{tabular}

